\title{
Impacto orçamentário na compra de equipamentos de proteção individual para enfrentamento da Covid-19
}

RESUMO | A Covid-19 é causada pelo coronavírus e transmitida por contato próximo, gotículas e aerossóis. Para minimizar o risco de transmissão, profissionais de saúde devem fazer uso de equipamentos de proteção individual, como máscaras ou respiradores, aventais e luvas. Objetivou-se analisar a quantidade consumida destes equipamentos de proteção individual antes e durante a pandemia e também o impacto orçamentário causado para sua aquisição. Estudo retrospectivo, compreendendo os meses de janeiro/fevereiro de 2020 (antes da pandemia Covid - 19) e março/abril (durante a pandemia Covid - 19), em um hospital público. Realizado avaliação do consumo e custo de aquisição dos insumos considerados equipamento de proteção individual.Os dados foram coletados em sistema de informação próprio da instituição. Todos os itens analisados apresentaram aumento importante na quantidade utilizada e, principalmente, no valor de compra, chegando custar $525 \%$ mais caro comparado aos meses sem pandemia. O aumento dos custos estava relacionado à escassez dos produtos tanto no mercado nacional, quanto internacional. Entender os valores pagos e estabelecer controle de dispensação dos equipamentos, favorece o planejamento orçamentário.

Palavras-chaves: Custos Hospitalares; Infecções por Coronavírus, Equipamento de Proteção Individual.

ABSTRACT | Covid-19 is caused by the coronavirus and transmitted by close contact, droplets and aerosols. To minimize the risk of transmission, healthcare professionals should use personal protective equipment, such as masks or respirators, aprons and gloves. The objective was to analyze the amount consumed of this personal protective equipment before and during the pandemic and also the budgetary impact caused for its acquisition. Retrospective study, comprising the months of January / February 2020 (before the Covid pandemic - 19) and March / April (during the Covid pandemic - 19), in a public hospital. Evaluation of consumption and cost of acquisition of inputs considered as personal protective equipment was performed. Data were collected in the institution's own information system. All the items analyzed showed an important increase in the quantity used and, mainly, in the purchase value, reaching $525 \%$ more expensive compared to the months without a pandemic. The increase in costs was related to the scarcity of products both in the national and international markets. Understanding the amounts paid and establishing equipment dispensing control, favors budgetary planning. Keywords: Hospital Costs; Coronavirus Infections; Personal Protective Equipment.

RESUMEN | El covid-19 es causado por el coronavirus y se transmite por contacto cercano, gotitas y aerosoles. Para minimizar el riesgo de transmisión, los profesionales de la salud deben usar equipo de protección personal, como mascarillas o respiradores, delantales y guantes. El objetivo fue analizar la cantidad consumida de este equipo de protección personal antes y durante la pandemia y también el impacto presupuestario que ocasionó su adquisición. Estudio retrospectivo, que comprende los meses de enero / febrero de 2020 (antes de la pandemia Covid - 19) y marzo / abril (durante la pandemia Covid - 19), en un hospital público. Se realizó una evaluación de consumo y costo de adquisición de insumos considerados como equipo de protección personal, los datos fueron recolectados en el sistema de información propio de la institución. Todos los artículos analizados mostraron un aumento importante en la cantidad utilizada y, principalmente, en el valor de compra, llegando a ser un 525\% más caro respecto a los meses sin pandemia. El aumento de costos estuvo relacionado con la escasez de productos tanto en el mercado nacional como internacional. Conocer los montos pagados y establecer el control de dispensación de equipos favorece la planificación presupuestaria.

Palabras claves: Costos de Hospital; Infecciones por Coronavirus; Equipo de Protección Personal.

\section{Karen Aline Batista da Silva}

Enfermeira. Doutora em Enfermagem pelo Departamento de Enfermagem da Faculdade de Medicina de Botucatu - Unesp. Diretora do Departamento de Logística de Atendimento do Hospital das Clínicas da Faculdade de Medicina de Botucatu.

ORCID: 0000-0002-8643-5333

\section{Patrícia Maria Moratelli Giuliani}

Farmacêutica. Especialista em Gestão Hospitalar. Gerente de Compras e Contratos do Hospital das Clínicas da Faculdade de Medicina de Botucatu.

ORCID: 0000-0002-2415-8799

Recebido em: 09/10/2020

Aprovado em: 04/11/2020

\section{Telma Aparecida de Camargo}

Enfermeira. Mestre em Enfermagem pelo Departamento de Enfermagem da Faculdade de Medicina de Botucatu - Unesp. Gerente de Materiais do Hospital das Clínicas da Faculdade de Medicina de Botucatu. ORCID: 0000-0002-9358-7175

\section{Karina Alexandra Batista da Silva Freitas}

Enfermeira. Mestre e Doutoranda em Enfermagem pelo Departamento de Enfermagem da Faculdade de Medicina de Botucatu - Unesp. Supervisora da Seção de Quimioterapia do Hospital das Clínicas da Faculdade de Medicina de Botucatu. ORCID: 0000-0002-3413-8468

\section{Ana Lúcia Gregório}

Enfermeira. Mestre em Enfermagem pelo Departamento de Enfermagem da Faculdade de Medicina de Botucatu - Unesp. Enfermeira do Núcleo de Padronização de Materiais do Hospital das Clínicas da Faculdade de Medicina de Botucatu. ORCID: 0000-0001-5764-0154

\section{Lis Amanda Ramos Toso}

Enfermeira. Especialista em Auditoria e Gestão Hospitalar. Enfermeira do Núcleo de Padronização de Materiais do Hospital das Clínicas da Faculdade de Medicina de Botucatu.

ORCID: 0000-0003-1734-569X 
INTRODUÇÃO

E m dezembro de 2019, um grupo de casos de pneumonia de origem desconhecida foi reportado em Wuhan, província de Hubei, China. O agente causador foi identificado como um novo coronavírus (SARS-CoV), causando a Covid-19, que desde então, infectou milhões de pessoas no mundo. A apresentação clínica pode variar de casos assintomáticos a pneumonia grave, com choque séptico e falência de múltiplos órgãos ${ }^{1}$.

O vírus é transmitido por contato próximo, gotículas e também aerossóis gerados durante a realização de procedimentos como intubação orotraqueal, ressuscitação cardiopulmonar, broncospia, etc. As precauções baseadas no modo de transmissão são exigidas a todos os profissionais de saúde a fim de prevenir a transmissão do vírus nos locais de assistência direta ao paciente ${ }^{2}$.

Otimizar os cuidados ofertados a pacientes, principalmente os que se encontram em estado grave, auxilia na redução de impactos nocivos à assistência ${ }^{3}$.

O Ministério do Trabalho e Emprego, por meio da Portaria N. ${ }^{\circ} 25$, DE 15 de outubro de 2001, NR 6, denomina equipamento de proteção individual (EPI) "todo dispositivo ou produto, de uso individual utilizado pelo trabalhador, destinado à proteção de riscos suscetíveis de ameaçar a segurança e a saúde no trabalho", devendo ser fornecido ao empregado, gratuitamente, pela empresa contratante ${ }^{4}$.

Para realizar a assistência aos casos suspeitos ou confirmados de infecção pelo novo coronavírus, a Anvisa publicou a nota técnica $n^{\circ}$ 04/2020, estabelecendo os EPIs necessários em cada situação ${ }^{5}$.

Os EPI, incluindo máscaras cirúrgicas, respiradores, luvas, aventais e protetores oculares, possuem papel primordial e indispensável na proteção dos profissionais de saúde, causando barreiras que podem evitar a infecção pelo coronavírus ${ }^{2,3}$. A utilização de EPI não evita acidentes e possíveis contaminações, mas minimi-

“

O vírus é transmitido por contato próximo, gotículas e também aerossóis gerados durante a realização de procedimentos como intubação orotraqueal, ressuscitação cardiopulmonar, broncospia, etc. As precauções baseadas no modo de transmissão são exigidas a todos os profissionais de saúde a fim de prevenir a transmissão do vírus nos locais de assistência direta ao paciente. zam as consequências dos acidentes de trabalho, com probabilidade de redução de danos ${ }^{6,7}$.

O uso racional e apropriado de EPI são estratégias que devem ser utilizadas para melhorar sua disponibilidade nas áreas assistenciais, frente ao desabastecimento global, com coordenação de mecanismos de gestão da cadeia de abastecimento e compra ${ }^{2}$.

Diante desta afirmação, planejar a compra e monitorar a dispensação e o uso de EPI pode impactar diretamente no custo do produto às instituições hospitalares, justificando o presente estudo, que objetivou analisar a quantidade consumida destes equipamentos antes e durante a pandemia e também o impacto orçamentário causado para sua aquisição.

\section{MÉTODO}

Estudo retrospectivo, de abordagem quantitativa, compreendendo os meses de janeiro/fevereiro de 2020 (antes da pandemia Covid - 19) e março/abril (durante a pandemia Covid - 19), em um hospital público de nível quaternário, localizado no interior do Estado de São Paulo, referência para atendimento de 68 municípios da região, abrangendo aproximadamente, 2 milhões de habitantes.

Realizado avaliação do consumo e custo de aquisição dos insumos considerados $\mathrm{EPI}$, agrupados em: máscara cirúrgica tripla e respirador N95 / PFF2, luvas descartáveis de procedimentos (não estéreis) e luvas cirúrgicas estéreis; aventais descartáveis.

Os dados referentes à quantidade de itens adquiridos, com seus respectivos valores, foram extraídos do sistema de Compras Extras (CE), exclusivo da instituição, e utilizado diariamente para visualização e alimentação de dados referentes às licitações eletrônicas e solicitações de compra direta. Possibilita a visualização da quantidade adquirida de cada item, valor, data de entrega, período de vigência de licitação, empenhos, etc.

Para analisar a dispensação de EPI foi 
utilizado o sistema informatizado Soul MV, módulo suprimentos, que comporta todas as entradas e saídas de insumos, peças, perecíveis, etc.

Após busca nos sistemas CE e MV os dados foram compilados em planilha Excel $®$, calculando a quantidade média de dispensação dos EPI por dia e por mês e o custo antes e durante a pandemia de Covid-19

\section{RESULTADOS}

A Tabela 1 demonstra o aumento crescente no uso de máscaras e respiradores no período analisado, acentuando-se nos meses de pandemia. O consumo médio diário de máscara tripla foi de 767 unidades no primeiro bimestre (antes da pandemia) e de 1284 unidades no segundo bimestre (durante a pandemia), e dos respiradores N95/PFF2, de 39 unidades e 106 unidades, respectivamente.

$\mathrm{O}$ aumento no consumo de respiradores N95/PFF2 nos meses de março e abril foi ocasionado pela obrigatoriedade do uso por toda equipe assistencial, sendo que antes eram exigidas somente em casos de atendimento a patologias infectocontagiosas com contaminação por aerossóis.

\begin{tabular}{lcccc}
$\begin{array}{l}\text { Tabela 1. Média do quantitativo (em unidades) } \\
\text { Brasil. } 2020\end{array}$ & de EPI utilizados/mês. Botucatu. \\
\hline EPI & 20.150 & 26.650 & 38.365 & 39.955 \\
\hline Cirúrgica cirúrgica tripla & 1.355 & 1.009 & 2.192 & 4.295 \\
\hline Respirador N95/PFF2 & 48.603 & 50.261 & 57.168 & 69.506 \\
\hline Avental descartável & 531.747 & 504.909 & 511.835 & 568.885 \\
\hline Luvas de procedimento (não estéreis) & 15.214 & 12.434 & 19.665 & 11.351 \\
\hline Luvas cirúrgicas estéreis & & & & Abril \\
\hline
\end{tabular}

Tabela 2. Valores pagos (em reais) por unidade de EPI antes e durante a pandemia por Covid - 19. Botucatu. Brasil. 2020

\begin{tabular}{lcc} 
EPI & Janeiro/Fevereiro & Março/Abril \\
Cirúrgica cirúrgica tripla & $\mathrm{R} \$ 0,09$ & $\mathrm{R} \$ 2,60$ \\
\hline Respirador N95/PFF2 & $\mathrm{R} \$ 1,45$ & $\mathrm{R} \$ 4,80$ \\
\hline Avental descartável & $\mathrm{R} \$ 1,70$ & $\mathrm{R} \$ 6,70$ \\
\hline Luvas de procedimento (não estéreis) & $\mathrm{R} \$ 0,17$ & $\mathrm{R} \$ 0,58$ \\
Luvas cirúrgicas estéreis & $\mathrm{R} \$ 0,72$ & $\mathrm{R} \$ 1,68$
\end{tabular}

Com relação aos aventais, é possível observar o aumento da quantidade utilizada por mês, perfazendo uma média diária de 1621 unidades no primeiro bimestre (antes da pandemia) e de 1906 unidades no segundo bimestre (durante a pandemia).

Para análise das luvas, foram consideradas as cirúrgicas estéreis e de procedimento (não estéreis) onde detectamos um aumento de consumo, porém, não na mesma proporção dos demais EPI. Isso se deve ao uso obrigatório de luvas para realização de procedimentos, sejam eles estéreis ou não, independente da patologia do paciente. $\mathrm{O}$ que não se aplicava aos aventais e máscaras, pois antes da pandemia por Covid-19, como protocolo, eram utilizados obrigatoriamente em isolamentos e em alguns procedimentos. A redução no número de luvas estéreis no mês de abril deve-se à suspensão das cirurgias eletivas, pois os leitos foram destinados à internação de pacientes suspeitos ou confirmados com Covid-19.

Em relação aos valores financeiros, a aquisição de máscara cirúrgica tripla sofreu um impacto ainda maior (Tabela 2), pois a empresa detentora do Registro 
O aumento do consumo de EPI pode acarretar impactos financeiros importantes nos orçamentos públicos devido escassez de desses produtos no mercado, ocasionando dificuldades em sua aquisição?.

Uma das causas da escassez de EPI no mercado é devido ao grande produtor mundial ser o epicentro da doença. A China teve a produção e venda dos EPI afetadas durante a pandemia, repercutindo no mercado mundial, mesmo quando retomaram às atividades normais 9 .

No início da pandemia, além da utilização de EPI por profissionais da saúde, a desinformação e pânico levaram à compra descontrolada pela população, também contribuindo para um desabastecimento ainda maior ${ }^{10}$.

Para atender os casos suspeitos ou confirmados com Covid-19, as instituições de saúde devem dispensar, diariamente, pelo menos, 25 unidades de aventais e máscaras cirúrgicas e 50 unidades de luvas de procedimento por paciente ${ }^{11}$.

O gerenciamento de EPI deve ser coordenado através de mecanismos básicos de gestão no fornecimento, considerando as previsões de uso baseadas na utilização racional dos itens solicitados; do estímulo ao uso de centralização no gerenciamento dos pedidos a fim de evitar duplicação de estoques; monitoramento e controle da distribuição dos EPI ${ }^{10}$.

Ainda há muitas dúvidas e debates em torno das medidas a serem tomadas pelos governantes a fim de minimizar o impacto econômico provocado pela pandemia. Independentemente da duração da pandemia, da crise e do grau de isolamento social imposto, os efeitos prejudiciais à economia terão magnitudes comparadas às maiores crises econômicas do mundo moderno, devido paralisação e desabastecimento das cadeias produtivas. Sendo as empresas elos da cadeia produtiva, a paralisação, ocasionada pelo isolamento social, impõe de forma crescente uma crise econômica desestruturante capaz de destruir mundialmente os elos produtivos ${ }^{12}$.
Paralelo à utilização de medidas de proteção, orientações devem ser estabelecidas a respeito do agir à medida que o cenário do trabalho evolui, mudando rapidamente e criando situações novas de risco, causando incertezas que necessitam ser manejadas ${ }^{3}$.

A utilização de EPI envolve o jeito certo e seguro de utilização durante paramentação e desparamentação, podendo surgir falhas individuais de trabalhadores que não aderem à forma segura e correta de agir. Entretanto, atribuir a contaminação do trabalhador a erros que possa ter cometido faz parte da cultura de culpabilização da vítima ${ }^{3}$, até porque seu uso apenas minimiza os efeitos ou consequências de um eventual acidente de trabalho ${ }^{13}$. As orientações corretas acerca da utilização do $\mathrm{EPI}$, bem como otimização de seu uso, devem ser fornecidas não somente diante de uma pandemia, mas como prática diária do ambiente assistencial.

A utilização do EPI é considerada como uma proteção ativa e dependente do comportamento individual, repetitivo e constante, é uma estratégia de prevenção com menos sucesso quando comparada à proteção passiva (coletiva), que assegura uma proteção relativamente automática ${ }^{14}$.

Em estudo desenvolvido na Atenção Primária à Saúde na cidade de Crato (CE), constatou um aumento no uso de EPI, mais expressivamente de máscaras cirúrgicas, causando colapso em seu fornecimento, que também pode ser justificado pela liberação de EPI aos profissionais de apoio, como auxiliares de serviços gerais, porteiros e agentes administrativos ${ }^{7}$. Essa informação difere da realidade de nosso estudo, já que os serviços de apoio são terceirizados e a responsabilidade de fornecimento de EPI aos colaboradores é de cada empresa contratada.

O reflexo do aumento no uso de EPI por profissionais da saúde, impactou na dificuldade de sua aquisição por municípios e estados brasileiros, já que necessitavam garantir medidas de biossegurança $\mathrm{a}^{13,15}$. 
Em Minas Gerais, o Conselho Municipal de Saúde recorreu aos órgãos fiscalizadores para denunciar os preços exorbitantes dos EPI. As máscaras cirúrgicas tiveram um aumento de $3.800 \%$ quando comparado com o período anterior à pandemia. O produto, que custava $\mathrm{R} \$ 0,10$ passou a custar $\mathrm{R} \$ 3,90$. O avental descartável acompanhou o aumento de preços e passou a custar R\$8, uma alta de $224,9 \%{ }^{16}$. Dados que corroboram com nossos resultados

As luvas de procedimentos (não estéreis) tiveram um aumento de $157 \%{ }^{16}$, sendo inferior aos dados encontrados em nosso estudo.

Uma das limitações do estudo foi a ausência, tanto no cenário nacional quanto internacional, de literatura disponível para comparação de custos e consumo de EPI.

\section{CONCLUSÃO}

O uso de EPI pela equipe assistencial é obrigatório e auxilia na prevenção da disseminação da Covid - 19. Diante isso, houve um aumento substancial no uso desses equipamentos, causando um impacto orçamentário significativo durante a pandemia, mesmo com a liberação extra de recursos pelo governo estadual.

A manutenção de EPI durante uma pandemia, deveria ser uma responsabilidade dos governos, tanto estadual como federal, em, além de distribuir às instituições públicas, controlar o aumento desenfreado e oportunista do mercado fornecedor. É compreensível que o aumento de preços no início da pandemia foi ocasionado pela escassez de matéria-prima, entretanto, após 7 meses, observa-se ainda um cenário de supervalorização nos preços dos EPI, sem perspectiva de melhora.

\section{Referências}

1. European Centre for Disease Prevention and Control (ECDC). Infection prevention and control for COVID-19 in healthcare settings. 2020. [acesso em 01 de ago. 2020]. Disponivel em: https://www.ecdc.europa.eu/sites/default/files/documents/COVID-19-infection-prevention-and-control-healthcare-settingsmarch-2020.pdf. 2. Organização Pan-americana da Saúde (OPAS). Uso racional de equipamentos de proteção individual para a doença causada pelo coronavírus 2019 (COVID-19) e considerações durante desabastecimentos graves de 06 de abr. 2020. [Orientação provisória]. [acesso em 01 de ago. 2020]. Disponível nível em: https://iris.paho. org/bitstream/handle/10665.2/52042/OPASBRACOVID1920045_por.pdf?sequence $=1$ \&isAllowed $=y$.

3. Almeida IM. Health protection for healthcare workers in COVID-19 times and responses to the pandemic. Rev Bras Saude Ocup. 2020;45:e17. https://doi.org/10.1590/ SCiELOPreprints. 140

4. Brasil. Ministério do Trabalho e Emprego. Norma Regulamentadora $n^{\circ} 6$, de 8 de junho de 1978: dispõe sobre os equipamentos de proteção individual (EPIs). Atualizada pela Portaria MTE/SIT n 194 de 7 de dezembro de 2010. Brasília; 2010. [acesso em 14 de mai. de 2020] Disponível em: http://trabalho.gov.br/images/Documentos/ SST/NR/nr-06-atualizada-2018.pdf.

5. Brasil. Agência Nacional de Vigilância Sanitária. Nota técnica 04/2020 - Orientações para serviços de saúde: medidas de prevenção e controle que devem ser adotadas durante a assistência aos casos suspeitos ou confirmados de infecção pelo novo coronavírus (SARS - CoV-2). Atualizada em 31 de mar. 2020. [acesso em 27 de jun. 2020]. Disponivel em: http://portal.anvisa.gov.br/documents/33852/271858/ Nota+T\%C3\%A9cnica+n+04-2020+GVIMS-GGTES-ANVISA/ab598660-3de4-4f14-8e6f-b9341c196b28.

6. World Health Organization (WHO). Coronavirus Disease 2019 (COVID-19): situation report [Internet]. Geneva:WHO; 2020; [acesso em 01 de ago]. Disponível em: https://apps.who.int/ iris/bitstream/handle/10665/332151/nCoVsitrep15May2020-eng. pdf? sequence= 1 \&isAllowed=y

7. Saraiva EMS et al. Impact of pandemia by Covid-19 on the provision of personal protection equipment. Braz. J. of Develop. 2020; 6(7): 43751-43762. DOI:10.34117/ bjdv6n7-115

8. Centers for Disease Control and Prevention (CDC). Pandemic Planning: Recom- mended Guidance for Extended Use and Limited Reuse of N95 Filtering Facepiece Respirators in Healthcare Settings. [acesso em 01 de ago. 2020]. Disponível em: https://www.cdc.gov/niosh/topics/hcwcontrols/recommendedguidanceextuse.html 9. Centers for Disease Control and Prevention. Factors to Consider When Planning to Purchase Respirators from Another Country. 2020 [cited 2020 May 11]. Available from: https://www.cdc.gov/coronavirus/2019-ncov/hcp/ppe-strategy/international-respirator-purchase.html

10. World Health Organization (WHO). Rational use of personal protective equipment (PPE) for coronavirus disease (COVID-19). [Internet]. 2020 [cited 2020 Apr 08]. Available from: https://apps.who.int/iris/bitstream/handle/10665/331498/WHO-2019-nCoV-IPCPPE_use-2020.2-eng.pdf

11. World Health Organization (WHO). Requirements and technical specifications of personal protective equipment (PPE) for the novel coronavirus (2019-ncov) in healthcare settings. [Internet]. 2020 [cited 2020 May 08]. Available from: https://iris. paho.org/bitstream/handle/10665.2/51906/requirements-\%20PPE-coronavirus-eng. pdf? sequence $=1$ \&isAllowed $=y$

12. Nogueira MO, Silva SP, Carvalho SS. Da virose biológica à virose econômica: uma vacina para microempresas no Brasil. Revista de Administração Pública. 2020; 54(4): 1010-1021.

13. Chan JF, et al. A familial cluster of pneumonia associated with the 2019 novel coronavirus indicating person-to-person transmission: a study of a family cluster. Lancet. 2020; 395(10223): 514-523. https://doi.org/10.1016/50140-6736(20)301549 14. Huang C; et al. Clinical features of patients infected with 2019 novel coronavirus in Wuhan, China. Lancet 2020; 395: 497-506. https://doi.org/10.1016/501406736(20)30183-5

15. Ferioli M, Cisternino C, Leo V, Pisani L, Palange P, Nava S. Protecting healthcare workers from SARS-CoV-2 infection: practical indications. Eur Respir Rev. 2020; 29(155):200068. doi: 10.1183/16000617.0068-2020

16. Portal G1. Com pandemia de coronavírus, equipamentos de proteção individual têm alta nos preços. [Internet] 2020 [cited 2020 oct 05]. Available:https://g1.globo. com/mg/minas-gerais/noticia/2020/05/22/com-pandemia-de-coronavirus-equipamentos-de-protecao-individual-tem-alta-nos-precos.ghtml 